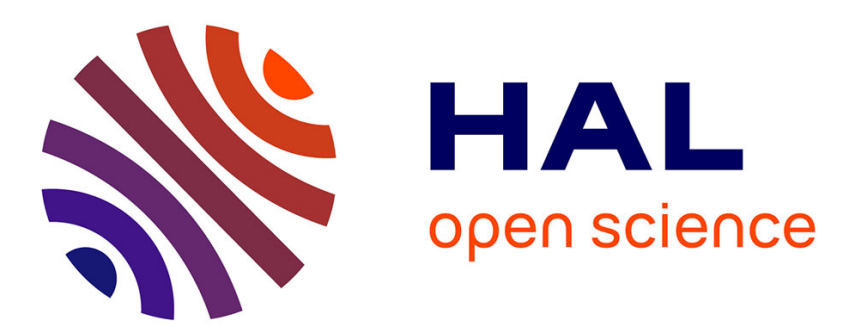

\title{
Etude expérimentale d'un récepteur solaire en nid d'abeilles pour le chauffage solaire des gaz à haute température
}

\author{
G. Olalde, J.L. Peube
}

\section{- To cite this version:}

G. Olalde, J.L. Peube. Etude expérimentale d'un récepteur solaire en nid d'abeilles pour le chauffage solaire des gaz à haute température. Revue de Physique Appliquée, 1982, 17 (9), pp.563-568. 10.1051/rphysap:01982001709056300 . jpa-00245032

\section{HAL Id: jpa-00245032 https://hal.science/jpa-00245032}

Submitted on 1 Jan 1982

HAL is a multi-disciplinary open access archive for the deposit and dissemination of scientific research documents, whether they are published or not. The documents may come from teaching and research institutions in France or abroad, or from public or private research centers.
L'archive ouverte pluridisciplinaire HAL, est destinée au dépôt et à la diffusion de documents scientifiques de niveau recherche, publiés ou non, émanant des établissements d'enseignement et de recherche français ou étrangers, des laboratoires publics ou privés. 


\title{
Etude expérimentale d'un récepteur solaire en nid d'abeilles pour le chauffage solaire des gaz à haute température
}

\author{
G. Olalde et J. L. Peube
}

Laboratoire d'Energétique Solaire, B.P. 5, Odeillo 66120 Font-Romeu, France

(Reçu le 24 septembre 1981, révisé le 26 février 1982, accepté le 29 mars 1982)

Résumé. - L'étude concerne l'utilisation des matériaux réfractaires à structure nid d'abeilles, comme récepteurs solaires fonctionnant à haute température. Un récepteur expérimental en CSi alvéolaire a été conçu et testé au four' solaire de $6,5 \mathrm{~kW}$ du Laboratoire d'Energétique Solaire à Odeillo. Les résultats expérimentaux ont montré la possibilité de chauffer de l'air à des niveaux de température compris entre $800 \mathrm{~K}$ et $1200 \mathrm{~K}$ avec une efficacité thermique variant entre $25 \%$ et $55 \%$.

\begin{abstract}
This study was concerned with the heating of a gas at high temperature by solar radiation through honeycomb material. An experimental honeycomb receiver was designed to be used with a $6.5 \mathrm{~kW}$ furnace of the Solar Energy Laboratory in Odeillo (France) and constructed. Temperature profile, gas temperature level and thermal efficiency of the receiver are reported in this paper.
\end{abstract}

Nomenclature.

$A=$ Surface de référence éclairée par le rayonnement incident.

$C_{\mathrm{g}}=$ Chaleur spécifique du gaz à pression constante.

$d=$ Diamètre d'une alvéole.

$G \quad=$ Débit massique.

$L^{+}=$Distance adimensionnelle de pénétration $L^{+}=L_{\mathrm{p}} / d$.

$L_{\mathrm{p}}=$ Distance de pénétration.

$T_{\mathrm{a}}=$ Température ambiante.

$T_{\mathrm{i}}=$ Température intermédiaire entre la matrice solide et le gaz, donnée par thermocouple.

$T_{0}=$ Température de la matrice solide sur la surface éclairée.

$X=$ Coordonnée axiale.

$X^{+}=$Coordonnée axiale adimensionnelle $X^{+}=X / d$.

$Y=$ Coordonnée radiale.

$\alpha_{M}=$ Facteur d'absorption de la matrice solide.

$\varepsilon_{M}=$ Facteur d'émission de la matrice solide.

$\varepsilon_{\mathrm{s}}=$ Facteur d'émission du matériau solide à l'état massif.

$\rho=$ Masse volumique du matériau alvéolaire.

$\sigma=$ Constante de Stefan.

$\phi_{0}=$ Flux incident.

$\phi_{\mathrm{R}}=$ Flux réfléchi.

$\phi_{\mathrm{E}}=$ Flux émis.

$\phi_{\mathrm{G}}=$ Flux évacué par le gaz.
$\phi_{\mathrm{C}}=$ Flux perdu par convection-conduction

$\phi_{\mathrm{r}}=$ Densité de flux.

$\theta_{0}=$ Température du gaz à la sortie du milieu.

$\theta_{\mathrm{a}}=$ Température du gaz à l'entrée.

$\eta_{\mathrm{g}}=$ Efficacité thermique.

$E^{+}=$Eclairement (unité arbitraire).

$\xi \quad=$ Porosité de la matrice alvéolaire.

Introduction. - Le chauffage solaire des gaz à haute température est intéressant, à cause de ses nombreuses applications. Citons par exemple :

- Dans le domaine de la thermochimie : les réactions solide-gaz [1] et les réactions en phase gazeuse [2].

- Dans le domaine de la thermodynamique : la conversion de l'énergie solaire en électricité au moyen des centrales héliothermoélectriques à haut rendement de conversion (Cycles Stirling, BraytonJoule).

Pour toutes ces applications apparaît la conception d'un composant clé et véritablement nouveau, où l'échange thermique est effectué : "le récepteur solaire ".

Le récepteur solaire placé au foyer d'un système de concentration est exposé à des cycles répétés et soumis à des contraintes thermiques très élevées.

Des études sur des récepteurs métalliques [3, 4] et en céramique [5] pouvant fournir un gaz sous pres- 
sion à haute température $(1000-1300 \mathrm{~K})$ ont été effectuées.

D'autres types de récepteurs solaires utilisant des milieux divisés ou poreux ont été étudiés $[1,6,7]$. Ceux-ci semblent particulièrement intéressants car les milieux poreux jouent un double rôle, d'absorbeur solaire et d'échangeur de chaleur.

C'est à cette dernière conception que peut se rattacher le récepteur en nid d'abeilles ou alvéolaire.

En effet, il offre une surface d'échange solide-gaz très importante $\left(1100 \mathrm{~m}^{2} / \mathrm{m}^{3}\right.$ pour un milieu alvéolaire formé de $11 \times 10^{5}$ alvéoles $\left./ \mathrm{m}^{2}\right)$. De plus, sa structure géométrique est un excellent piège pour le rayonnement.

DISPOSITIF RÉALISÉ. - Le système expérimental est représenté schématiquement sur la figure 1.

1.1.1 Récepteur. - Le matériau en nid d'abeilles utilisé lors de nos expériences est en CSi.

Le tableau I donne quelques caractéristiques physiques de ce matériau.

Fig. 1. - Schéma expérimental.

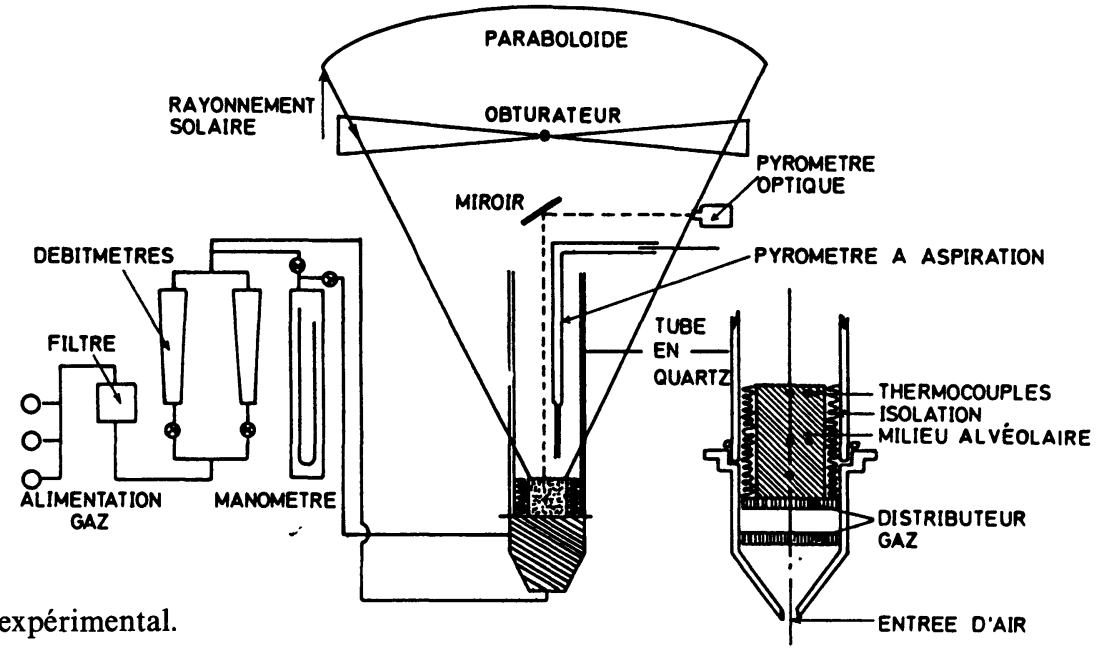

[Schematic diagram of experimental unit.]

Tableau I.

\begin{tabular}{|l|c|c|c|c|c|c|}
\hline Nature & $\begin{array}{c}\text { Surface de } \\
\text { contact }\end{array}$ & $d$ & $\begin{array}{c}\rho \text { du nid } \\
\text { d'abeilles }\end{array}$ & $\frac{\text { Alvéoles }}{\mathrm{m}^{2}}$ & $\begin{array}{c}\text { Température } \\
\text { maximale } \\
\text { d'utilisation }\end{array}$ & $\xi$ \\
\hline $\mathrm{CSi}\left({ }^{*}\right)$ & $1100 \mathrm{~m}^{2} / \mathrm{m}^{3}$ & $3 \times 10^{-3} \mathrm{~m}$ & $400-500 \mathrm{~kg} / \mathrm{m}^{3}$ & 110000 & $1640 \mathrm{~K}$ & 0,7 \\
\hline
\end{tabular}

(*) Spectramic TM Honeycomb (NORTON).

Un cylindre en alumine (diamètre intérieur $0,060 \mathrm{~m}$, hauteur $0,15 \mathrm{~m}$ ), contient le matériau alvéolaire; les dimensions de l'échantillon testé (Fig. 2) sont : diamètre $0,060 \mathrm{~m}$, hauteur $0,075 \mathrm{~m}$.

Pour éliminer les échanges thermiques avec l'extérieur, ce cylindre est isolé par une fibre silico-alumineuse. Huit thermocouples sont placés dans le matériau alvéolaire à l'intérieur d'un volume de référence (voir Annexe I).

Le cylindre calorifugé contenant le nid d'abeille est placé à l'intérieur d'un cylindre en quartz. Le gaz (air) est introduit dans la partie inférieure du récepteur à travers un diffuseur et il circule en sens inverse du flux d'énergie.

1.1.2 Concentrateur. - Le dispositif optique utilisé est le concentrateur solaire de $6,5 \mathrm{~kW}$ du laboratoire d'Energétique Solaire à Odeillo 8 .

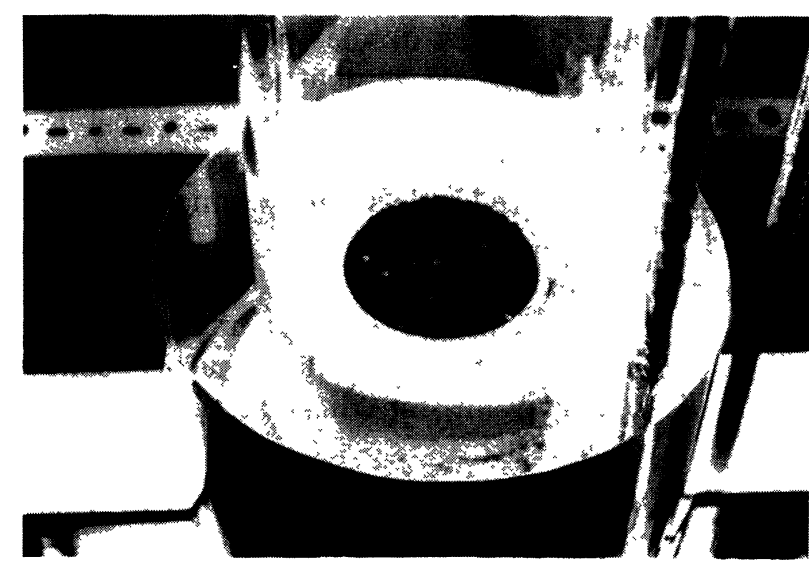

Fig. 2. - Récepteur solaire alvéolaire en CSi.

[CSI honeycomb solar receiver.] 
Celui-ci est constitué de 306 miroirs hexagonaux (rayon de courbure 7,60 m), épousant la forme d'une calotte sphérique. L'axe de l'installation est vertical. Un obturateur à pales radiales placé entre le flux réfléchi et le plan focal, permet de moduler la puissance au foyer. La figure 3 présente la répartition énergétique sur un diamètre du plan focal.

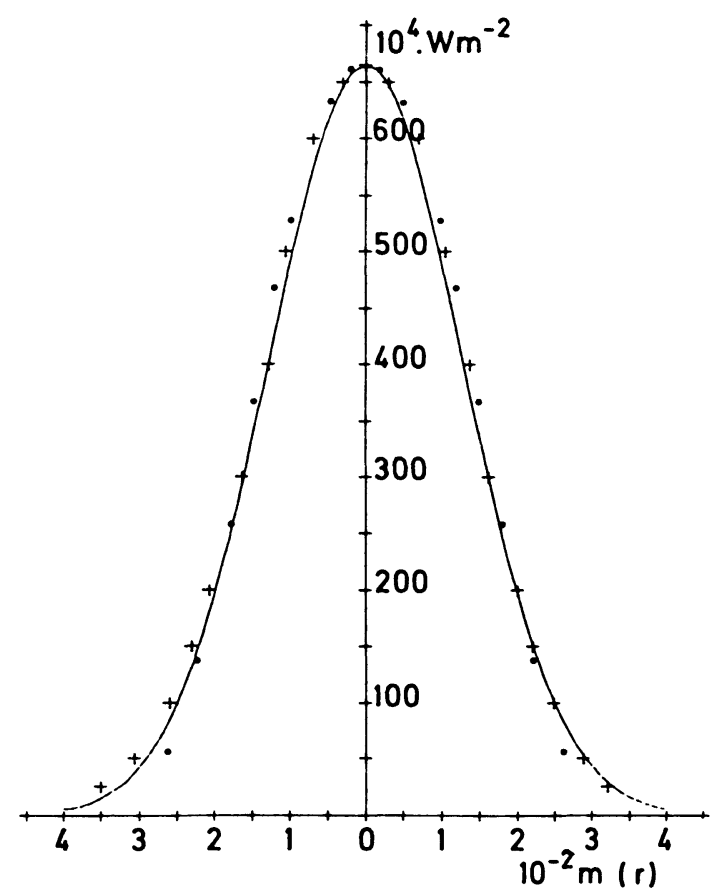

Fig. 3. - Répartition expérimentale de l'éclairement énergétique sur un diamètre E.O. du plan focal.

[Experimental flux density distribution along the E.O. axis in the focal plane of the solar concentrator.]

1.2 SySTÈMES DE MESURES. - Les mesures de température ont été réalisées :

- Par des thermocouples; ce sont des câbles "Thermocoax » de diamètre extérieur $0,001 \mathrm{~m}$ composés de conducteurs chromel-alumel, isolés l'un de l'autre ainsi que de la gaine de protection en inconel par un oxyde réfractaire pulvérulent. Ils sont directement placés dans les alvéoles à trois niveaux différents $(0,010 \mathrm{~m}, 0,025 \mathrm{~m}$ et $0,050 \mathrm{~m}$ à partir de la face exposée au rayonnement) suivant l'axe du récepteur. La valeur de la température donnée par des thermocouples est une valeur intermédiaire entre celle du gaz et celle de la matrice solide.

- Par pyrométrie optique pour la détermination de la température de la matrice alvéolaire frappée par le rayonnement solaire concentré.

- Par un pyromètre à aspiration; pour effectuer la mesure de la température du gaz sortant du matériau alvéolaire nous avons réalisé un pyromètre à aspiration. Il est constitue d'un thermocouple Pt-Pt Rh $10 \%$ placé sur l'axe d'un système de deux écrans

ReVUe de PHYSIQUE APPLlQUiE. I. 17, N" 9, StPILMBRE 1982 réfléchissants en alumine à travers lesquels le gaz circule à grande vitesse.

Le débit du gaz (air) est contrôlé par un débitmètre à cônes (du type rotamètre) associé à un régulateur de pression. La température du gaz à l'entrée du récepteur est la température ambiante.

Pour déterminer l'atténuation du rayonnement solaire lorsqu'il pénètre à l'intérieur du matériau en nid d'abeilles, nous avons évalué la distance adimensionnelle de pénétration du rayonnement " $L^{+}$». Elle est définie comme le rapport entre la distance de pénétration du rayonnement solaire dans la matrice alvéolaire et le diamètre d'une alvéole $L^{+}=L_{\mathrm{p}} / d$.

L'évaluation de ce paramètre nous permet de connaître la distance à partir de laquelle le rayonnement concentré qui frappe la face exposée au rayonnement solaire est absorbé. Une fibre optique en quartz (de diamètre 0,000 $4 \mathrm{~m}$ ) gainée par un tube en alumine à parois minces est couplée à une photo-résistance et permet de mesurer l'atténuation du rayonnement solaire dans la matrice. On introduit une extrémité de la fibre optique à l'intérieur de la matrice alvéolaire à l'aide d'un dispositif permettant son déplacement axial tandis que l'autre extrémité est couplée à la photo-résistance délivrant ainsi un signal proportionnel à l'éclairement reçu.

2. Bilan énergétique. - Nous supposons :

- le flux incident $\phi_{0}$ uniforme,

- le récepteur à l'équilibre thermique.

L'expression théorique du bilan énergétique global s'écrit donc :

$$
\begin{aligned}
\phi_{0}=\phi_{\mathrm{R}}+\phi_{\mathrm{E}}+\phi_{\mathrm{G}}+ & \phi_{\mathrm{C}} \\
\phi_{0}=A\left(1-\alpha_{\mathrm{M}}\right) \phi_{0}+ & A \varepsilon_{\mathrm{M}} \sigma\left(T_{0}^{4}-T_{\mathrm{a}}^{4}\right)+ \\
& +A G C_{\mathrm{g}}\left(\theta_{0}-\theta_{\mathrm{a}}\right)+\phi_{\mathrm{c}} .
\end{aligned}
$$

L'évaluation des termes radiatifs entrant dans le bilan ne peut être qu'approximative en raison de la difficulté d'évaluation des propriétés radiatives du système. Celles-ci dépendent à la fois des propriétés intrinsèques des matériaux et des grandeurs liées au milieu alvéolaire qu'ils constituent.

Les facteurs $\alpha_{M}$ et $\varepsilon_{M}$ ont été évalués à l'aide de l'expression suivante :

$$
\varepsilon_{M}=\varepsilon_{\mathrm{S}} /\left[(1-\xi)\left(1-\varepsilon_{\mathrm{S}}\right)+\varepsilon_{\mathrm{S}}\right] .
$$

Cette équation est utilisée dans la plupart des études concernant la détermination des propriétés radiatives des milieux poreux en faisant les hypothèses d'un facteur d'émission égal à 1 pour les pores et que la matrice solide est "grise " $[12,13,7]$.

La valeur calculée à partir de cette équation pour des milieux ayant une porosité de l'ordre de 0,5 correspond à $5 \%$ près, à la valeur expérimentale.

La répartition non uniforme de l'éclairement sur la face avant du milieu poreux est pratiquement 
inévitable, et ce d'autant plus que la densité de flux recherchée est grande, c'est-à-dire que l'on s'approche du foyer.

Les erreurs commises dans l'évaluation des termes radiatifs seraient donc très importantes si on supposait toute la surface éclairée à une température moyenne $T_{0}$ (effet du terme de rayonnement en $T^{4}$ ).

Pour les minimiser, nous avons choisi un volume d'étude significatif du transfert de chaleur (matrice solide-gaz) à haute température mais dans lequel la variation de la densité de flux est faible (voir Annexe I).

L'efficacité thermique du récepteur est calculée par rapport à la puissance évacuée par le gaz. Elle est définie par l'expression :

$$
\eta_{\mathrm{g}}=\phi_{\mathrm{G}} / \phi_{0} .
$$

3. Résultats. - Comme nous l'avons mis en évidence la valeur de la température donnée par les thermocouples à l'intérieur du matériau alvéolaire est une valeur intermédiaire entre celle de la matrice solide et celle du gaz, ce qui nous conduit à présenter dans nos résultats un profil axial de température intermédiaire $T_{\mathrm{i}}$.

Nous avons porté sur la figure 4 l'évolution du profil axial de température $T_{\mathrm{i}}$ en fonction de $X$ pour différentes valeurs de débits massiques. La figure 5 montre l'évolution de la température de sortie de l'air $\theta_{0}$ en fonction du débit massique. On remarque que la température de sortie de l'air diminue lorsque le débit augmente.

La distance à laquelle le rayonnement solaire est arrêté dans le matériau alvéolaire est fonction de l'angle d'incidence, des propriétés radiatives et des caractéristiques géométriques de la matrice solide.

La figure 6 fait apparaître l'atténuation de l'éclairement $E^{+}$(unité arbitraire) le long de l'axe, en fonction de $X^{+}$. La figure 7 représente le profil radial de la distance de pénétration $L^{+}$dans le récepteur et montre

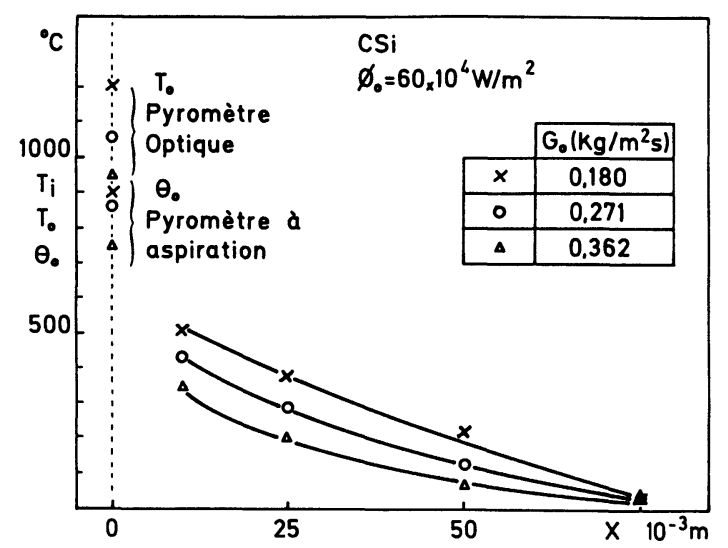

Fig. 4. - Profil axial de température $T_{\mathrm{i}}$ en fonction de $X$ pour différentes valeurs de $G_{0}$.

[Température profile $T_{\mathrm{i}}$ as function of $X$ for three different values of $G_{0}$.]

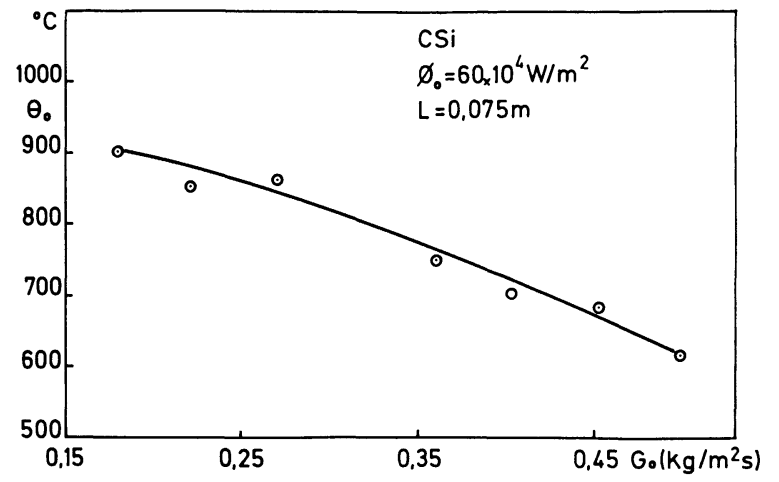

Fig. 5. - Evaluation de la température de sortie de l'air en fonction de $G_{0}$.

[Gas outlet temperature vs. $G_{0}$.]

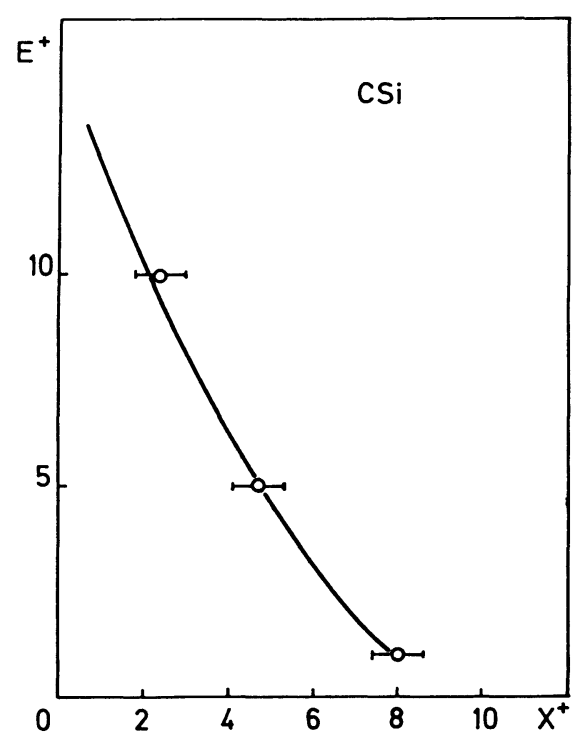

Fig. 6. - Eclairement $E^{+}$(unité arbitraire) à l'intérieur du milieu alvéolaire en fonction de $X^{+}$.

[Flux density $E^{+}$as function of $X^{+}$into the CSi honeycomb material.]

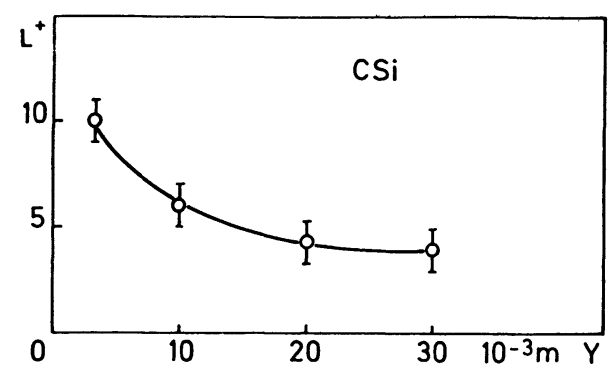

Fig. 7. - Profil radial de la distance de pénétration du rayonnement solaire dans le milieu alvéolaire en $\mathrm{CSi}$.

[Radial profile of the penetration of solar radiation into the $\mathrm{CSi}$ honeycomb material.] 
que cette distance diminue lorsqu'on s'éloigne de l'axe de symétrie.

L'efficacité thermique $\eta_{\mathrm{g}}$ du récepteur varie entre $25 \%$ et $55 \%$. La figure 8 montre cette variation en fonction du débit massique. L'analyse de chaque terme du bilan énergétique (1) est représentée en figure 9 sous la forme de répartition en pourcentage de la puissance totale $\phi_{0}$ reçue sur le récepteur.

On y montre les résultats les plus représentatifs de l'ensemble des essais.

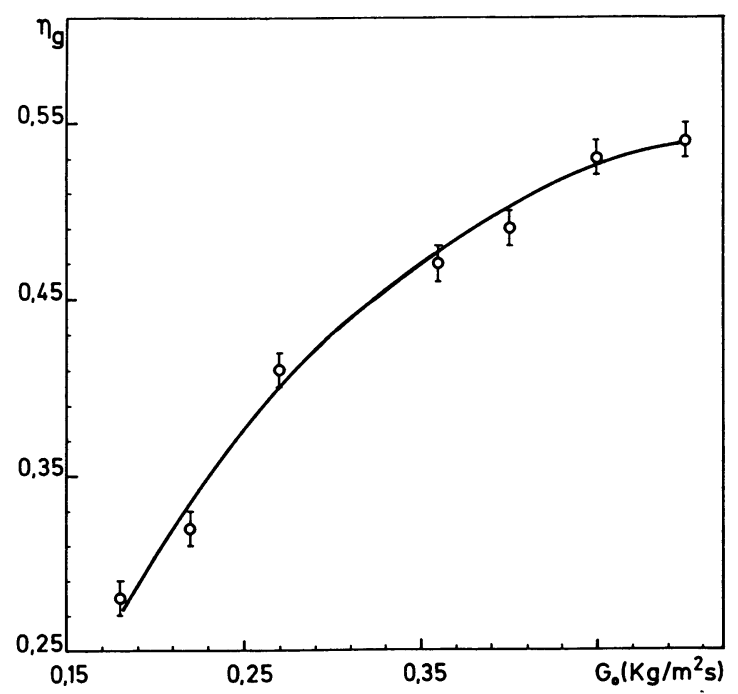

Fig. 8. - Représentation schématique du bilan énergétique du récepteur.

[Schematic representation of the energy balance of the receiver.]

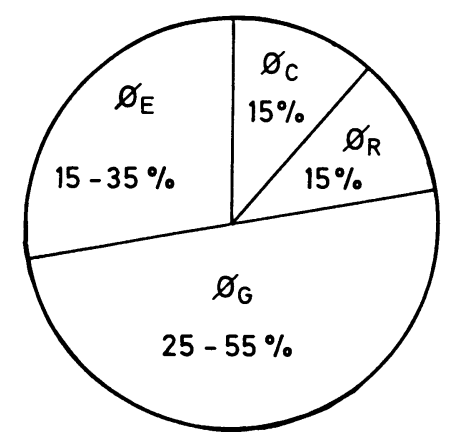

Fig. 9. - Bilan énergétique.

[Energy balance.]

4. Conclusion. - La mise en œuvre des matériaux réfractaires à structure alvéolaire ou en nid d'abeilles comme récepteur solaire s'avère très intéressante pour le chauffage des gaz à haute température.

Des niveaux de température compris entre $800 \mathrm{~K}$ et $1200 \mathrm{~K}$ ont été atteints pour un éclairement solaire de $60 \times 10^{4} \mathrm{~W} / \mathrm{m}^{2}$. Dans ces conditions, l'efficacité thermique du récepteur varie entre $25 \%$ et $55 \%$.
Ces résultats ont été obtenus pour un récepteur à configuration plane. Une grande amélioration peut être espérée pour des configurations en forme de cavité car ces dernières réduisent les pertes radiatives du système [9].

Par ailleurs, il a été mis en évidence que la pénétration du rayonnement améliore les performances des récepteurs à milieux divisés $[10,11]$. Le développement de nouveaux matériaux alvéolaires mettant à profit ce phénomène est une autre voie de recherche visant à l'amélioration de l'efficacité de ces absorbeurs.

Grâce à l'importante surface d'échange et à la résistance mécanique et chimique (haute résistance à l'oxydation) des récepteurs alvéolaires, on peut envisager de les utiliser comme échangeurs de chaleur pour des applications non solaires, par exemple dans le domaine de la récupération à haute température de la chaleur d'origine industrielle.

Annexe I. - Détermination du volume de référence. En vue de minimiser les effets radiaux ainsi que les erreurs commises dans l'évaluation des termes radiatifs de l'équation (1) nous avons défini, un volume de référence figure A.1. La variation de la densité de flux $\phi_{\mathrm{r}}$ sur la face éclairée de ce volume est faible. La plupart de

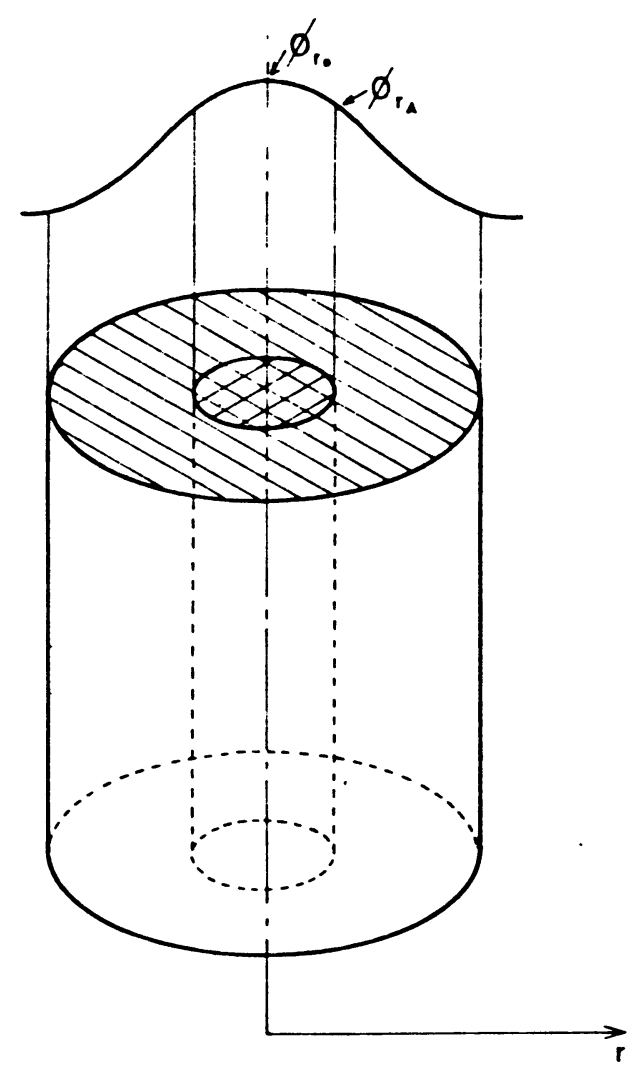

Fig. A.1. - Représentation du volume de référence pour le bilan énergétique global.

[Schematic of reference volume used to calculate thermal efficiency.] 
nos mesures ont été réalisées dans un rapport $\phi_{\mathrm{rA}} / \phi_{\mathrm{r} 0}=0,8$. La répartition de la densité de flux au foyer dans nos expériences est donnée par l'expression suivante :

$$
\phi_{\mathrm{r}}=I_{1} \exp \left(-I_{2} r^{2}\right)
$$

avec

$$
\begin{aligned}
& I_{1}=665 \mathrm{~W} / \mathrm{cm}^{2} \\
& I_{2}=0,303 \mathrm{~cm}^{-2}
\end{aligned}
$$

Le diamètre du volume de référence est

$$
d_{\mathrm{VR}}=2\left[\frac{\ln 665-\ln 0,80 \phi_{\mathrm{r} 0}}{0,303}\right]^{1 / 2}
$$

La puissance reçue sur la surface éclairée du volume de référence est obtenue en intégrant l'équation (A.1)

$$
\phi_{\mathrm{Tr}}=\Pi \frac{I_{1}}{I_{2}}\left[1-\exp \left(-I_{2} \frac{d_{\mathrm{VR}}^{2}}{4}\right)\right] .
$$

\section{Bibliographie}

[1] Flamant, G., Hernandez, D., Bonet, C., Traverse, J. P., « Experimental aspects of the thermochemical conversion of solar energy decarbonation of $\mathrm{CaCO}_{3}$ ». Sol. Energy 4 (1980) 385-395.

[2] ChubB, T. A., "Analysis of gaz dissociation solar thermal power system ». Sol. Energy 17 (1975) 129-136.

[3] Gintz, J., Bartlett, D., Zentmer, R., «1MWth bench moded solar receiver test program $»$. Revue Phys. Appl. 15 (1980) 363-368.

[4] Wintrebert, G., Blay, D., Ortiz, F., « Etude d'un récepteur solaire à haute température pour cycle thermodynamique à gaz ". Systèmes solaires thermodynamiques 15-20 juin (1980) Marseille, France (Ed. C.N.R.S.) pp. 405-410.

[5] Jarvinen, P. O., "Solar heated air receivers ». Sol. Energy 19 (1977) 139-147.

[6] HunT, A. J., "A new solar thermal receiver utilizing a small particle heat exchanger $"$. Proc. 14th I.E.C.E.C. Boston 5-10 August (1979).

[7] Olalde, G., "Etude théorique et expérimentale du chauffage d'un gaz s'écoulant à travers un matériau poreux soumis au rayonnement solaire concentré ". Thèse de Docteur ès-Sciences Physiques (19 février 1981), Université de Perpignan.
[8] Arnaud, G., Flamant, G., Olalde, F., Robert, J. F., "Les fours solaires de recherche du Laboratoire d'Energétique Solaire d'Odeillo ». Entropie No 97 (1981) pp. 139-146.

[9] Gennevieve, F., Olalde, G., Daguenet, M., « Chauffage solaire des gaz », Revue Phys. Appl. 15 (1980) 985-994.

[10] Flamant, G., «Etudes théorique et expérimentale d'un récepteur solaire à lit fluidisé ". Systèmes solaires thermodynamiques 15-20 juin (1980) Marseille, France (Ed. C.N.R.S.) pp. 495-501.

[11] Cabannes, F., « Echauffement d'un gaz par un rayonnement solaire concentré dans un matériau poreux. Calcul de l'influence de la pénétration du rayonnement dans le matériau ». Rev. Hautes Tempér. Réfract. Fr. (à paraître).

[12] Hernandez, D., "Contribution à la mise au point de méthodes de mesure pour la détermination de la température d'une particule en mouvement et d'un lit fluidisé solaire ". Thèse de $3 \mathrm{e}$ cycle, Université de Perpignan (déc. 1978).

[13] Flamant, G., « Thermochimie solaire : Etude de procédés, application à la décarbonation de la calcite ॥. Thèse de Docteur-Ingénieur, Université Paul Sabatier, Toulouse (octobre 1978). 